\title{
Performance of the HADES-TOF RPC wall in a Au-Au beam
}

\author{
A. Blanco* \\ LIP-Laboratório de Instrumentação e Física Experimental de Partículas, Coimbra, Portugal \\ E-mail: alberto@coimbra.lip.pt \\ P. Fonte \\ LIP-Laboratório de Instrumentação e Física Experimental de Partículas, Coimbra, Portugal \\ ISEC-Instituto Superior de Engenharia de Coimbra, Coimbra, Portugal \\ E-mail: fontedcoimbra.lip.pt
}

\section{Lopes}

LIP-Laboratório de Instrumentação e Física Experimental de Partículas, Coimbra, Portugal E-mail: luisalbertodcoimbra.lip.pt

\section{W. Koenig}

GSI Helmholtzzentrum für Schwerionenforschung GmbH, Darmstadt, Germany

E-mail: W.Koenig@gsi.de

\section{J. A. Garzon}

LabCAF, Universidade de Santiago de Compostela, USC, Santiago de Compostela, Spain E-mail: juanantonio.garzon@usc.es

\section{G. Kornakov}

LabCAF, Universidade de Santiago de Compostela, USC, Santiago de Compostela, Spain E-mail: kornakov@gmail.com

In this work we present results concerning in beam operation of the new Resistive Plate Chamber (RPC) Time of Flight (TOF) wall for the High-Acceptance DiElectron Spectrometer (HADES) spectrometer at GSI.

The new RPC-TOF wall, fully integrated in the spectrometer, has been successfully operated under final conditions corresponding to collision of $\mathrm{Au}+\mathrm{AU} @ 1.25 \mathrm{GeV}$.

Results confirm an uniform average time response below 80 ps $\sigma$ along with an average longitudinal position resolution better than $12 \mathrm{~mm} \sigma$. The multihit capability of the system has also been investigated showing a slight degradation of time resolution when particles hit the detector at neighbouring cells, which demonstrates the multihit capability of the concept (individually electrically shielded RPCs).

XI workshop on Resistive Plate Chambers and Related Detectors - RPC2012,

February 5-10, 2012

INFN Laboratori Nazionali di Frascati Italy

\footnotetext{
${ }^{*}$ Speaker.
} 


\section{Introduction}

HADES (High-Acceptance DiElectron Spectrometer) at GSI-SIS [1] is a second-generation dielectron detector, operates in the energy regime of $1-2 \mathrm{AGeV}$ and consists of a 6-coil toroidal magnet centered on the beam axis and six identical detection sections located between the coils and covering polar angles between $18^{\circ}$ and $85^{\circ}$. Its main aim is to explore the phase diagram of nuclear matter at moderate temperatures and nuclear densities, a key problem in heavy-ion physics, where modifications of the particle masses are predicted due to the partial restoration of the chiral symmetry of Quantum Chromodinamics (QCD) [2].

HADES has launched its experimental program and meanwhile confirmed ([3], [4]) the results obtained by the DiLepton Spectrometer (DLS) collaboration for light systems [5]. For this purpose, HADES was equipped with a large Time of Flight (TOF) Wall made of plastic scintillator bars readout by photomultipliers. However the multiplicity requirements, to be able to explore for the first time in the energy regime of a few $\mathrm{AGeV}$ the heavy $\mathrm{Au}-\mathrm{Au}$ systems in the dielectron channel, called for an upgraded TOF detector, which was already foreseen [6], with higher granularity. A solution for the HADES Inner TOF Wall based on shielded timing Resistive Plate Chambers (RPCs) [7] was installed in the spectrometer in 2010 and commissioned during 2011. Here, we present intrinsic performance results for the RPC-TOF from data taking during 2011 under final conditions corresponding to collision of $\mathrm{Au}+\mathrm{AU} @ 1.25 \mathrm{GeV}$.

\section{The RPC-TOF wall}

The RPC-TOF wall is divided in 6 sectors (or sextants) of trapezoidal shape, named as $S_{i}$ with $i=1 \ldots 6$, covering a total area of around $8 \mathrm{~m}^{2}$ (fig. 1). It is composed by 1116 strip-like, 4-gap, symmetric, timing RPCs [8] ("cells"). Each cell is is individually shielded for robust multi-hit performance, a key characteristic of the design as will be shown later. The cells inside of each sector are organized in two partially overlapping layers. Each layer is composed by 31 rows and 3 columns, ranging the widths between 2.2 and $5 \mathrm{~cm}$ and the lengths between 12 and $52 \mathrm{~cm}$. The cells are made of 3 aluminium electrodes and 2 glass (soda-lime) electrodes, all of $2 \mathrm{~mm}$ thickness. The 4 gaps are defined by PEEK ${ }^{1}$ monofilaments of $0.270 \mathrm{~mm}$ diameter. The ensemble is housed inside individual shielding tubes. A comprehensive description of the RPC-TOF wall can be found in [9].

The RPC-TOF wall is operated with a gas mixture composed of $90 \% \mathrm{C}_{2} \mathrm{H}_{2} \mathrm{~F}_{4}$ and $10 \% \mathrm{SF}_{6}$ under a continuous gas flow of $30 \mathrm{~cm}^{3} / \mathrm{min}$ (per sector) at a nominal High Voltage (HV) of $5500 \mathrm{~V}$ together with the different final subsystems, namely: Front End Electronics (FEE) [10], Data Acquisistion System (DAQ) [11], Low Voltage (LV) [12], Slow Control (SC) [13] and HV power supply (CAEN A1526).

\section{Timing performance}

\subsection{Intrinsic time resolution}

The intrinsic time resolution is calculated for those particles with hits in overlapping cells by

\footnotetext{
${ }^{1}$ Polyetheretherketone
} 


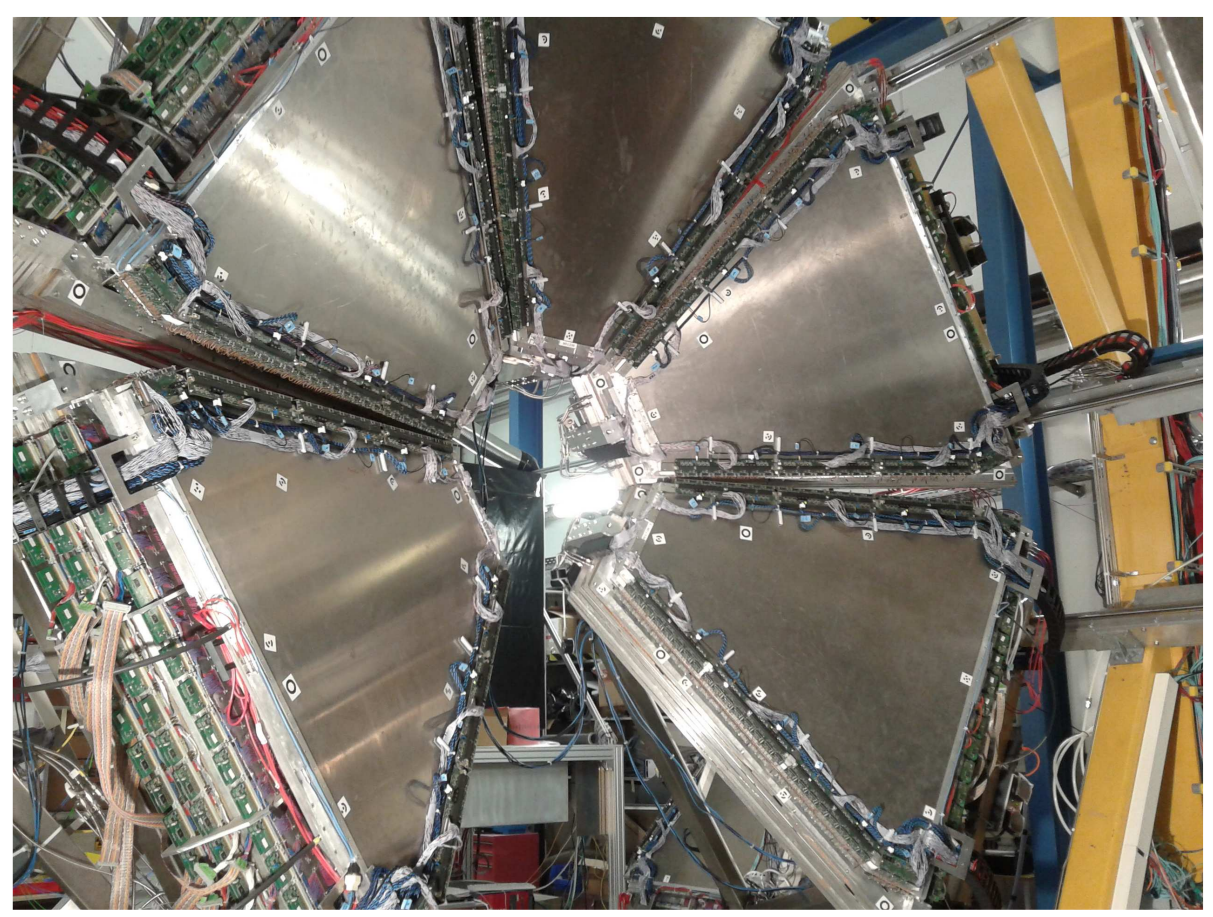

Figure 1: RPC-TOF wall divided in 6 sectors (lower most sector (4) is missed in the picture) covering a total area of around $8 \mathrm{~m}^{2}$.

characterizing the distribution:

$$
\Delta T_{\text {up-down }}=\frac{1}{2}\left[\left.\left(t_{l}+t_{r}\right)\right|_{u p}-\left.\left(t_{l}+t_{r}\right)\right|_{\text {down }}\right]
$$

where $t_{l}$ and $t_{r}$ are the measured times left and right for each cell and up and down denote the two overlapping cells on different layers. In order to characterize the resulting non Gaussian distribution, the $\sigma$ of a Gaussian fit within $\pm 1.5 \sigma$ about the mean of the original distribution, $\left.\sigma \Delta T_{\text {up-down }}\right|_{ \pm 1.5 \sigma}$ was calculated. Assuming equal resolution for both cells, the intrinsic time resolution for a single cell (after corrected by charge and longitudinal position, see [14]) is $\sigma_{t}=\left(\left.\sigma \Delta T_{\text {up-down }}\right|_{ \pm 1.5 \sigma}\right) / \sqrt{2}$.

It should be noticed that no particle selection is performed in this analysis and therefore any type of particle fulfilling the condition of producing hits in overlapping cell is analysed.

Fig. 2.a shows $\sigma_{t}$ for overlapping cells in the same row for the entire chain (cell + FEE + DAQ), as a function of row number for all sectors. All active channels have a resolution under $100 \mathrm{ps}$ (time resolution requirement), without noticeable correlation with cell position. The average intrinsic time resolution for all sector, calculated as the mean of a Gaussian fit to the distribution of individual $\sigma_{t}$, are 81, 80, $80,79,82,78$ ps for $S_{1}, S_{2}, S_{3}, S_{4}, S_{5}$ and $S_{6}$ respectively and globally (for all sectors) $80 \mathrm{ps}$, see fig $2 . \mathrm{b}$.

\subsection{Multihit time resolution}

The same analysis described in Sec. 3.1 was repeated for a restricted set of data, in which it was required that each primary hit (two hits in overlapping cells) had at least one another hit, 
a)
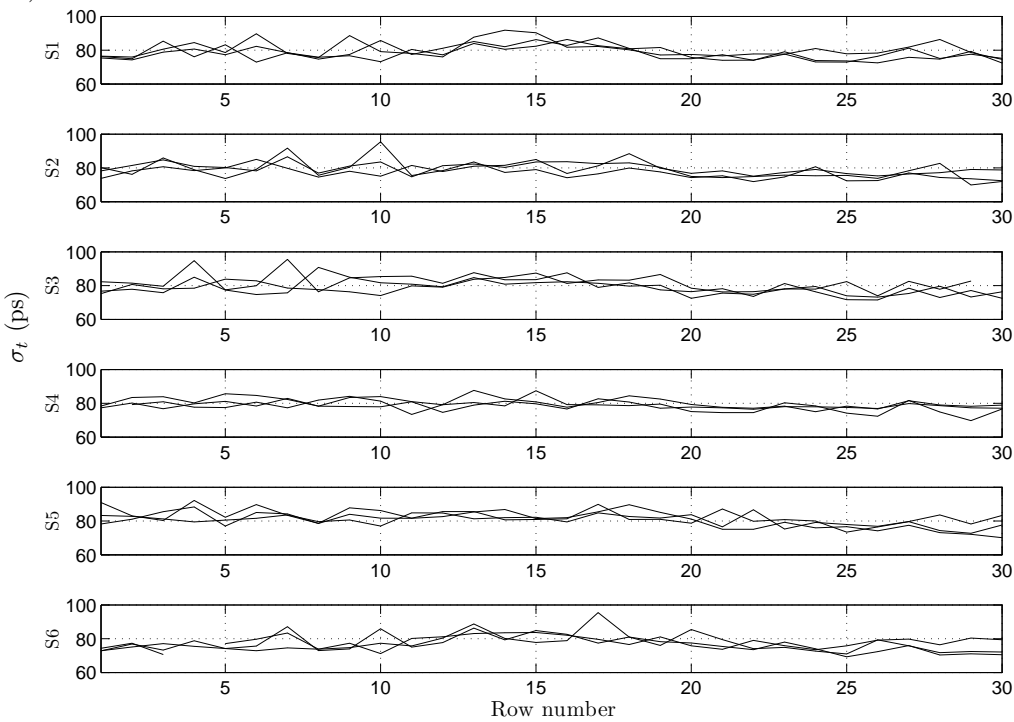

b)

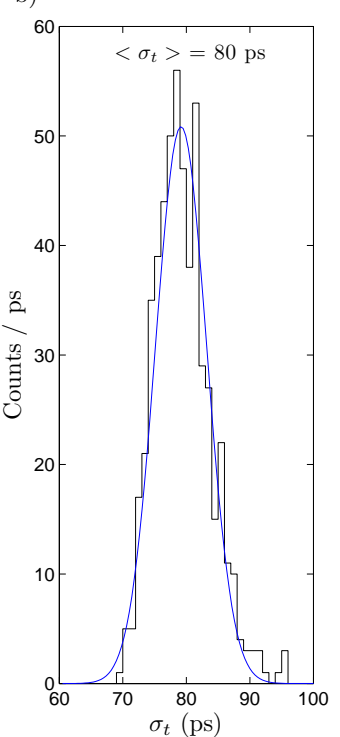

Figure 2: a) Intrinsic time resolution for overlapping cells in the same row for the entire chain (cell $+\mathrm{FEE}+$ DAQ), as a function of row number for all sectors. The average intrinsic time resolutions are 81, 80, 80 ,79, 82,78 ps for $S_{1}, S_{2}, S_{3}, S_{4}, S_{5}$ and $S_{6}$ respectively. b) Average intrinsic time resolution for all sectors 80 ps.

secondary, at a certain distance $D$ (this distance refers to rows). So, $D=0$ means that each primary hit in a given row has at least other secondary hit in the same row (each row has six cells). $D=1$ means that each primary hit in a given row, $n$, has at least one other secondary hit in row number $n-1$ or $n+1$ and no secondary hits are present in row $n$, etc.

Fig. 3.a illustrates for $\mathrm{S}_{2}$, the intrinsic time resolution for two different values of $D, 0$ and 4 . Additionally, it is also compared with result previously shown in Sec. 3.1 for all events $(D=X)$. Time resolution is clearly degraded when the situation of $D=4$ is compared with the result corresponding to the analysis of all events and even more degraded when compared with the scenario of $D=0$. Fig. 3.b, which shows $\sigma_{t}$ as a function of $D$ for $\mathrm{S}_{2}$, quantify this degradation. The average intrinsic time resolution degrades up to a value of 85 ps when at least a secondary hit is in the proximity of the primary hit (same row, $D=0$ ) and it is improved slightly in the scenario of $(D=1)$ down to $77 \mathrm{ps}$, when compared to the situation in which all events are analysed (80 ps). In the rest of scenarios $(D>1)$, the average time resolution is basically unaffected by the presence of a secondary hit and shows an average intrinsic resolution of around $71 \mathrm{ps}$. This figure demonstrates the multihit nature of the $\mathrm{Au}+\mathrm{Au}$ collisions, since the average distance $D$ to the secondary hit in the situation in which all events are analysed is around 0.6. It also shows the multihit capability of the RPC-TOF able to measure two neighbour hits with a slight degradation of the time resolution of a few ps.

\section{Position resolution}

The intrinsic longitudinal position resolution is calculated for those particles with hits in over- 

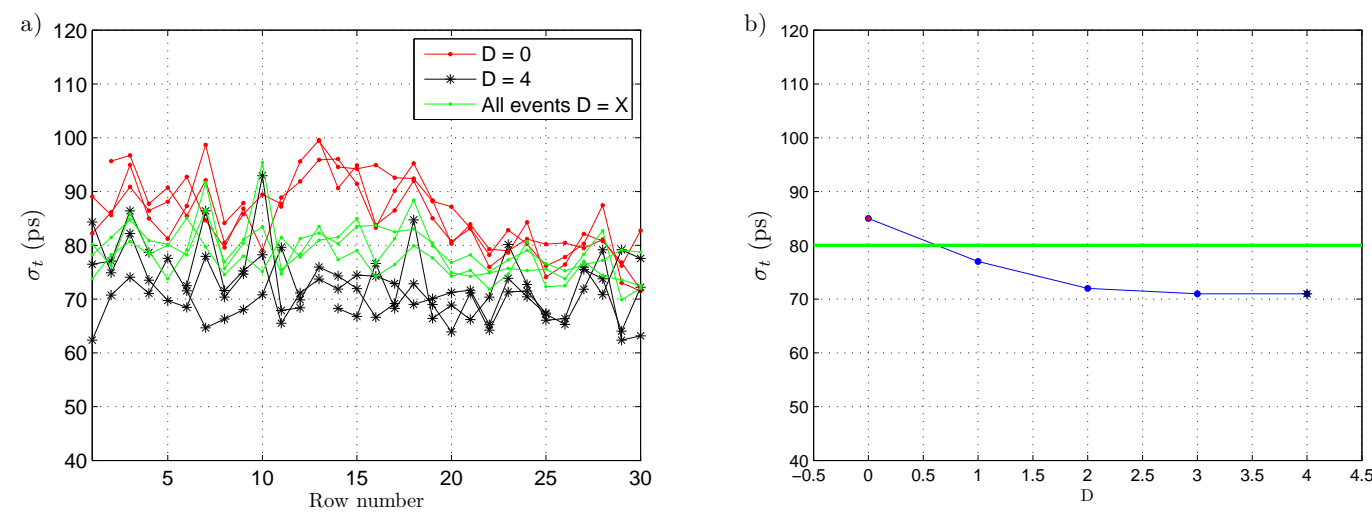

Figure 3: a) Illustration of the intrinsic time resolution for two different values of $D$ (distance in rows between the primary and secondary hits, see text), 0 and 4 and also the result for all events $(D=X)$. b) Average intrinsic time resolution as a function of $D$. This figure shows the multihit nature of the $\mathrm{Au}+\mathrm{Au}$ collisions, since the average distance $D$ to the secondary hit in the situation in which all events are analysed is around 0.6. It also shows the multihit capability of the RPC-TOF able to measure to neighbour hits with a slight degradation of a few $\mathrm{ps}$.

lapping cells by obtaining the $\sigma$ of the longitudinal positions difference distribution:

$$
\Delta X_{\text {up-down }}=\frac{v_{\text {prop }}}{2}\left[\left.\left(t_{l}-t_{r}\right)\right|_{\text {up }}-\left.\left(t_{l}-t_{r}\right)\right|_{\text {down }}\right]
$$

where $v_{\text {prop }}$ is the signal propagation velocity in the cell, $177 \mathrm{~mm} / \mathrm{ns}$ [14]. Assuming equal resolution for both cells, the position resolution for a single cell is $\sigma_{x}=\sigma\left(\Delta X_{\text {up-down }}\right) / \sqrt{2}$.

Fig. 4 shows $\sigma_{x}$ for overlapping cells in the same row, as a function of row number for all sectors. All active channels have a resolution under $12 \mathrm{~mm}$, without noticeable correlation with cell position. The average intrinsic position resolution for all sectors, calculated as the mean of a Gaussian fit to the distribution of individual $\sigma_{x}$, are 8.7, 8.9, 9.1 ,8.8, 8.1,8.6 mm for $\mathrm{S}_{1}, \mathrm{~S}_{2}, \mathrm{~S}_{3}$, $\mathrm{S}_{4}, \mathrm{~S}_{5}$ and $\mathrm{S}_{6}$ respectively and globally (for all sectors) $8.9 \mathrm{~mm}$, see fig $4 . b$.

\section{Conclusion}

The six sectors of the new HADES-TOF wall based on RPCs were successfully operated under final conditions corresponding to collision of Au + AU @1.25 GeV. Results confirm an uniform average time response below $80 \mathrm{ps} \sigma$ along with an average longitudinal position resolution better than $12 \mathrm{~mm} \sigma$. The multihit capability of the system has also been investigated. It shows a slight degradation of time resolution when particles hit the detector at neighbouring cells, demonstrating the multihit capability of the concept (individually electrically shielded RPCs).

\section{Acknowledgements}

This work was supported by FCT and FEDER/COMPETE under the contracts CERN/FP/109373/2009 and PTDC/FIS/11339/2009. 
a)
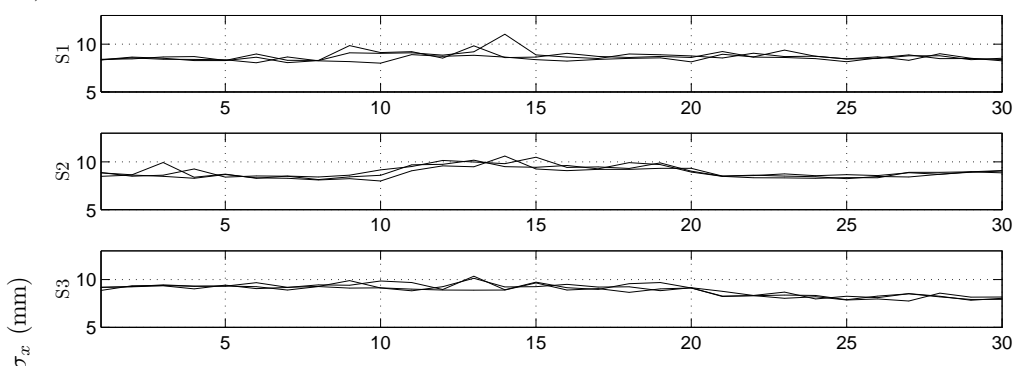$$
\text { 药 }
$$
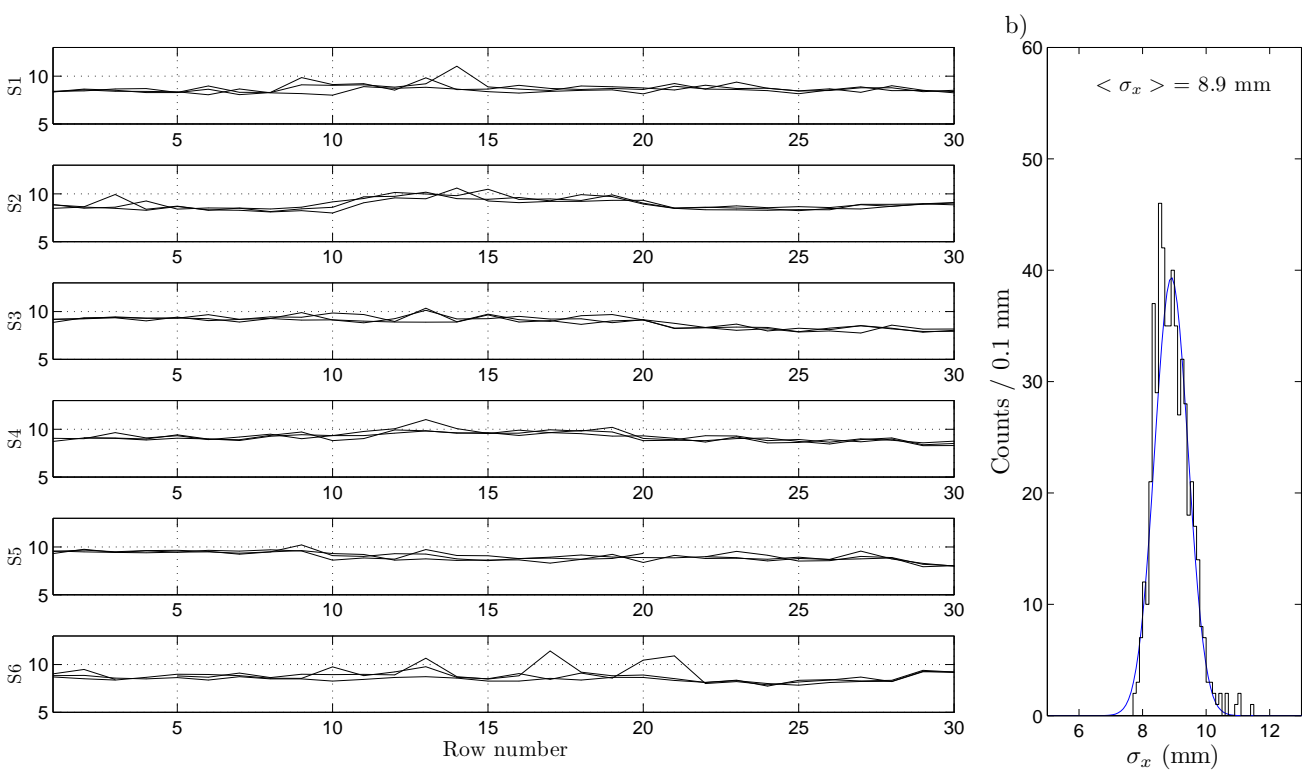

Figure 4: Intrinsic longitudinal position resolution for overlapping cells in the same row, as a function of row number for all sectors. The average intrinsic longitudinal position resolutions are 8.7, 8.9, 9.1 ,8.8, 8.1 ,8.6 $\mathrm{mm}$ for $\mathrm{S}_{1}, \mathrm{~S}_{2}, \mathrm{~S}_{3}, \mathrm{~S}_{4}, \mathrm{~S}_{5}$ and $\mathrm{S}_{6}$ respectively. b) Average intrinsic longitudinal position resolution for all sectors $8.9 \mathrm{~mm}$.

\section{References}

[1] P. Salabura et al., Nucl. Phys. A 749 (2005) 150.

[2] G. E. Brown and M. Rho, Phys. Rev. Lett. 66 (1991) 2720.

[3] G. Agakichiev et al., Phys. Rev. Lett. 98 (2007) 052302.

[4] R. Holzmann et al., Phys. Lett. B 663 /1-2 43.

[5] R. J. Porter et al., Phys. Rev. Lett. 79 (1997) 1229.

[6] P. Salabura et al., Eur. Phys. J. A 41 (2009) 243-277.

[7] C. Finck,et al., Nucl. Instr. Meth. A 508 (2003) 63.

[8] P. Fonte et al., Nucl. Instr. Meth. A 443 (2000) 201.

[9] D. Belver et al., Nucl. Instr. Meth. A 602 (2009) 687.

[10] D. Belver et al., IEEE TNS, Vol. 57, No. 5, October 2010, 2848.

[11] J. Michel et al., IEEE TNS, Vol. 58, No. 4, August 2011, 1745.

[12] A. Gil et al., EEE TNS, Vol. 56, No. 2, April 2009, 382.

[13] A. Gil et al., Nucl. Instr. Meth. A 661 (2012) S118.

[14] A. Blanco et al., Nucl. Instr. Meth. A 602 (2009) 691. 